\section{PROBING THE LIQUID-SOLID INTERFACE WITH DYNAMIC FORCE MICROSCOPY}

Stuart Lindsay, Arizona State University*

Judy Zhu, Molecular Imaging Corporation

*stuart.lindsay@asu.edu

\section{Summary}

Processes at real interfaces, such as drug dissolution, corrosion, enzyme activity and surfactant structure can now be studied with nanometer resolution using dynamic force microscopy. Optimal sensitivity is achieved when the tip is excited by direct application of a driving force using magnetic methods.

\section{Introduction}

The atomic force microscope is capable of imaging processes at nanometer resolution in fluids (Drake, Prater et al. 1989 ) and this has led to revolutionary developments in fields as diverse as biology, mineral formation, surfactant structures and electrochemistry. Contact mode operation, in which a force-sensing cantilever is lowered into contact with the surface and then swept across it while maintaining constant deflection, is unsuitable for many studies of this nature because of the ease with which fragile hydrated materials are disrupted by the probe.

The dynamic force microscope (DFM) is a much gentler interfacial probe because of its differential sensitivity. In DFM (Figure 1) the scanning probe is driven into oscillation and the absolute amplitude of the ac component of the deflection signal is recorded via the change in angular-position of a laser beam reflected from the oscillating cantilever. The technique is inherently sensitive to the liquid-solid interface, as we shall show as follows, and it is free from the damaging effects of drift in the absolute (dc) position of the probe. It offers several other advantages, among them improved rejection of low frequency (I/f) noise, less shearing of adsorbed samples and better imaging of viscoelastic materials which can appear substantially stiffer at higher frequencies.

There are two common methods for implementing DFM in fluids. In one, either the tip or the sample is vibrated by the ap-

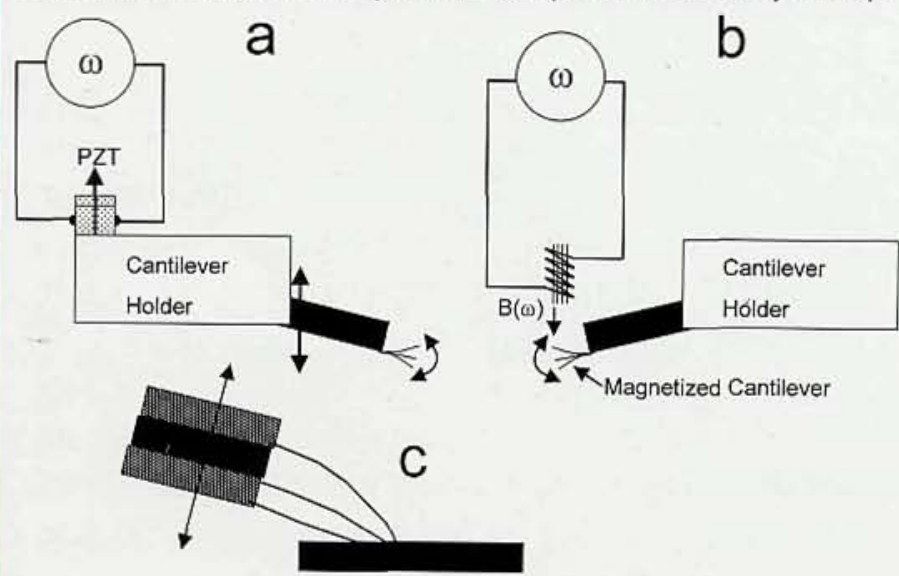

Figure 1: (a) Acoustically driven dynamic force microscope; a piezo-electric drive. A piezoelectric crystal (PZT) generates sound waves which displace the base of the cantilever driving the tip into oscillation when the driving frequency is near a bending resonance of the cantilever. A laser beam reflected off the end of the cantilever (not shown) senses a reduction in an amplitude on approach to the surface. (b) Magnetically driven dynamic force microscope. A solenoid drives a magnetic tip into bending without motion of the cantilever base. Consequently residual bending of the cantilever on contact with the surface is minimal. In contrast, relatively large motions of the cantilever base for the case of acoustic drive result in large residual bending signal when the cantilever touches the sample (c). plication of an acoustic excitation (Hansma, Cleveland et al. 1994; Lantz, O'Shea et al. 1994; Putman, Werf et al. 1994). This is illustrated in Figure 1a. In another, the tip is manipulated by a magnetic force, applied through the action of a magnetic field on a magnetic tip (Florin, Radmacher et al. 1993; Lindsay, Lyubchenko et al. 1993; Lantz, O'Shea et al. 1994; Han, Lindsay et al. 1996). In this article, we will outline the difference in operation between magnetic and acoustic DFM, review the imaging mechanism and illustrate the capabilities with examples of biological and pharmaceutical materials imaged in solution and controlled humidity.

\section{Magnetic vs. Acoustic DFM}

Acoustic drive is achieved with low frequency excitation $(\mathrm{kHz}$ to $\mathrm{MHz}$ ) which generates long wavelength modes that serve to translate the cantilever. These do not, of themselves, give rise to a detectable signal unless the cantilever is driven into bending motion. This occurs near mechanical resonances of the cantilever, when the tip moves more than the base of the cantilever. This distinction is of little consequence for operation in air, because the mechanical Q-factor (ratio of amplitude on resonance to driving amplitude) of the cantilever is high. In fluid, the motion of the tip is severely-damped, and the mechanical $Q$ factor drops to a small number, typically 3 . Thus, if the tip is to be driven at $3 \mathrm{~nm}$ peak amplitude, the cantilever holder must be displaced by $1 \mathrm{~nm}$. Such large amplitude displacements occur only at mechanical resonances of the microscope, so tip motion is excited only at these frequencies. On the other hand, magnetic drive bends the tip directly so that the response follows the intrinsic mechanical resonance of the cantilever itself. This is illustrated in Figure 2 which shows (a) the response of a magnetically-driven cantilever in water (along with the calculated response as a solid line) and (b) the measured response of an acoustically-driven cantilever (Han, Lindsay et al. 1996; Schaffer, Cleveland et al. 1996). Thus, acoustic drive is limited to frequencies close to a coincidence of accidental resonances in the microscope and the mechanical resonance of the cantilever itself.

A more serious problem is presented by the intrinsic background signal present with acoustic drive, as illustrated in Figure 1c. Because of the low mechanical $Q$, the cantilever base must be moved a large distance in acoustic drive, so that when the cantilever tip touches the surface, a significant residual bending signal is obtained (Figure 1c). In magnetic drive, the base remains fixed, so that the only motion on contact comes from residual magnetic forces on the cantilever, and, with appropriate construction, these can be negligible. The result is that the feedback signal (oscillation amplitude) falls to zero with magnetic drive. It does not in the case

\section{Continued on page 14}
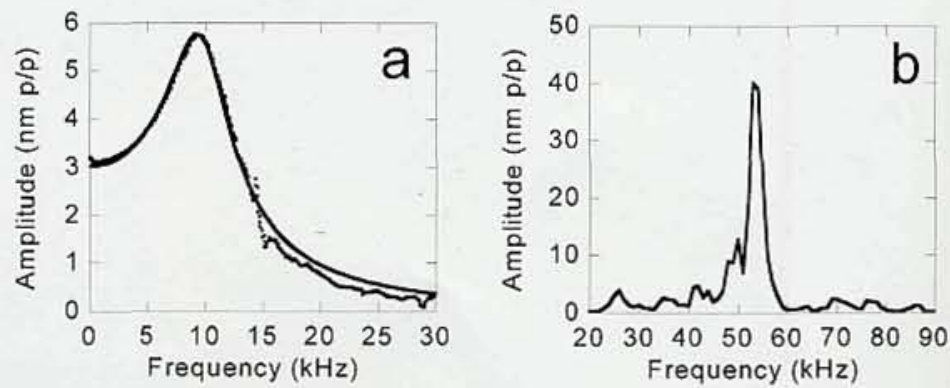

Figure 2: Amplitude of oscillation vs. drive frequency for (a) magnetic drive and (b) acoustic drive in water. The magnetic cantilever had a natural resonance near $8 \mathrm{kHz}$ and the overall response is close to that expected for an appropriately-damped cantilever (solid line). The cantilever used in the acoustic example had a natural frequency near $38 \mathrm{kHz}$, but the peak response occurs near $50 \mathrm{kHz}$ as a consequence of the vibrational properties of the microscope body. 


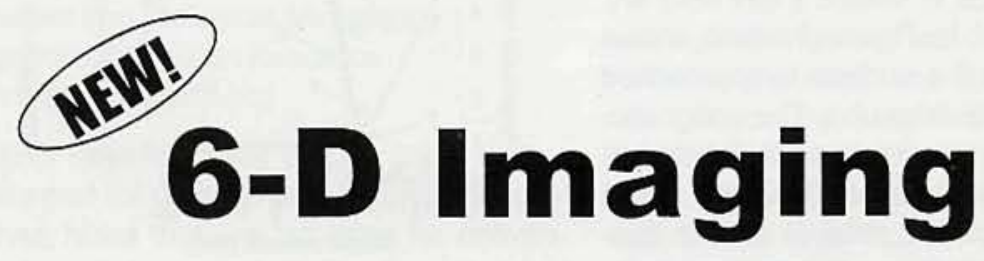

You've Got to See This

www.image1.com

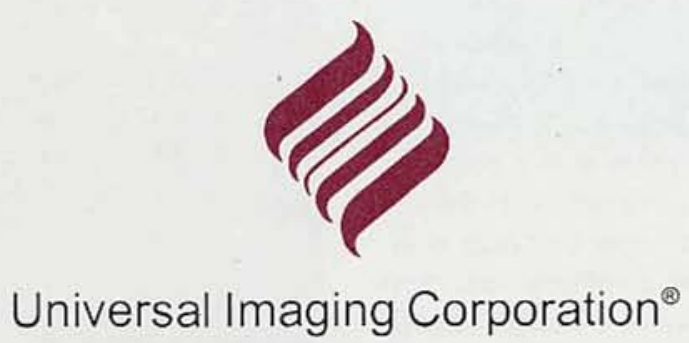

C 1999 Universal Imaging Corporation 


\section{PROBING THE LIQUID-SOLID INTERFACE WITH DYNAMIC FORCE MICROSCOPY Continued from page 12}

of acoustic drive. This is illustrated in Figure 3 which shows plots of the oscillation amplitude as the surface is approached for magnetic drive (a) and acoustic drive (b). The magneticdrive results in a signal that falls to zero on contact (labeled 3 ) whereas the acoustic drive does not (labeled 7) (Lantz, Liu et al. 1998). The indirect nature of acoustic drive is further illustrated by its behavior far from resonance. At low frequency, very little bending signal is induced, however, as the tip is pressed into the surface, the base continues to move, with the result that the bending signal actually increases on contact, shown by the dashed curve in Figure $3 \mathrm{~b}$.

\section{Non-contact operation by DFM in Fluid}

O'Shea et al. (O'Shea, Lantz et al. 1998) and Lantz et al. (Lantz, Liu et al. 1998) have shown that DFM in fluid can be operated in non-contact mode, utilizing the changes in fluid properties that extend some nanometers from a solid surface. Both groups used magnetic drive in order to control the amplitude and approach to the surface, free from the complications of acoustic background. In the case of organic fluids, a rapid increase in viscosity some distance from the interface causes the oscillation amplitude to be damped (O'Shea, Lantz et al. 1998). In the case of water, a stiffening occurs which results in a smaller oscillation amplitude at a given driving force (Lantz, Liu et al. 1998). This regime is shown in Figure 3a. As the surface is approached, the amplitude falls (labeled 1) but not by as much as the tip is approached (i.e., the gradient, $d A / d z$ is less than one). On hard contact with the underlying surface (labeled 2) the amplitude falls by as much as the tip is approached ( $d A$ ) $d z=1$ ). This condition holds only well below a resonant frequency of the tip as explained by Lantz et al. (Lantz, Liu et al. 1998) The same authors also give a simple formula which can be used to extract the interfacial stiffness as a function of distance from approach curves like these. Figure $3 a$ also shows the average dc deflection signal (thin lines). This signal does not rise until hard contact with the surface. In this example, the tip was pushed hard into the surface (not shown) so that on retraction (thin dotted line) the tip stuck to the surface (labeled 5). The corresponding ac signal (thick dashed line) remained at zero until the tip jumped off the surface (labeled 6). Note that the ac signal is only about $50 \%$ of its full value at this point, an indication of the noncontact nature of the interaction in the region labeled 1. For a full analysis, see the paper by Lantz et al. (Lantz, Liu et al. 1998). Operated at small amplitude reduction in water, the DFM probes contours of constant interfacial stiffness, sensing the underlying surface though its effect on the intervening fluid layer.

\section{Resolution in DFM}

The atomic force microscope has achieved the highest resolution in contact mode, where there is a possibility that an atomic-scale asperity can interact with features on the surface via short range interactions. This comes at the price of strong interactions between the tip and the sample, so, on biological materials, the best images have been made from densely packed films stuck strongly to an underlying substrate. This is not an optimal arrangement for the study of processes, but resolution in acoustic DFM has typically been limited to $10 \mathrm{~nm}$ or so. Examples of higher resolution have been shown on se-

Continued on page 16
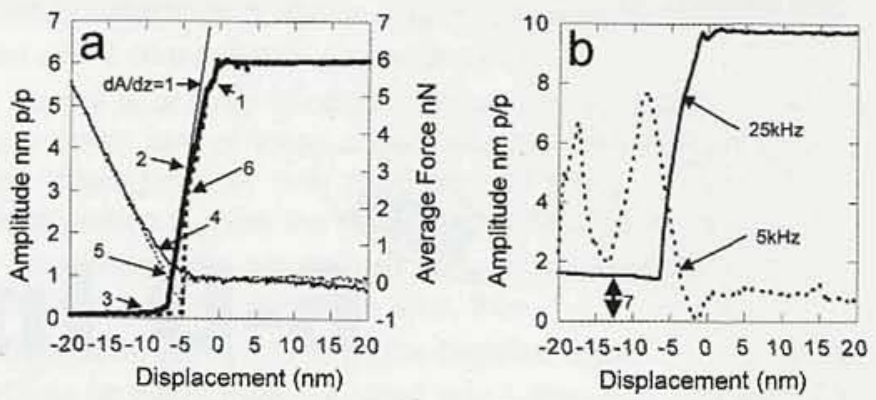

Figure 3: Approach curves showing oscillation amplitude vs. distance from an underlying surface for (a) magnetic DFM and (b) acoustic DFM. In (a) the approach curve is the heavy solid line, and the amplitude on retraction is shown by the heavy dashed line. The fine solid line shows the average displacement of the cantilever (calibrated in units of contact force in $\mathrm{nN}$ ) on approach. The fine dashed line is the same signal on retraction. The numbers refer to the regions of operation described in the text. Acoustic drive (b) results in a finite background on contact when driven near resonance (labeled 7) and an inverted polarity of control signal at low frequency (dashed line).

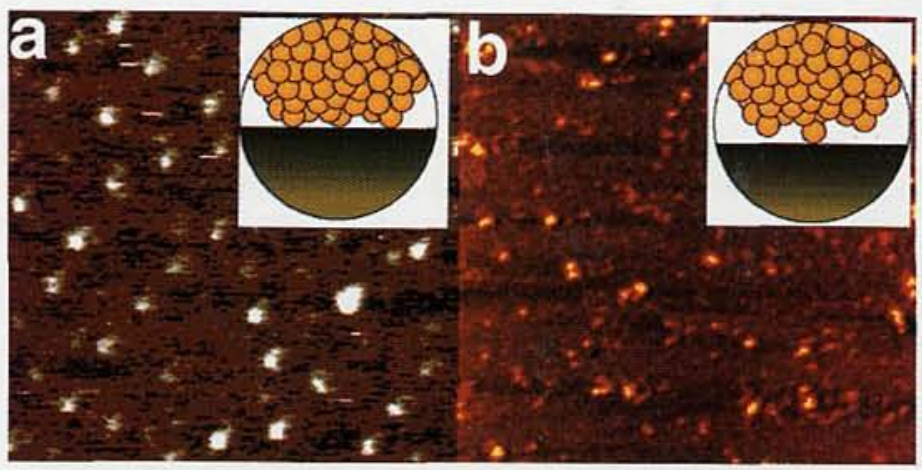

Figure 4: Images of cytochrome $c$ on graphite underwater taken with acoustic drive (a) and magnetic drive (b). The scales in both images are the same. Insets show how compression of asperities on a tip can result in lower resolution when the tip is approached to the surface roughly. (Sample courtesy of N.J. Tao of Florida International University.)

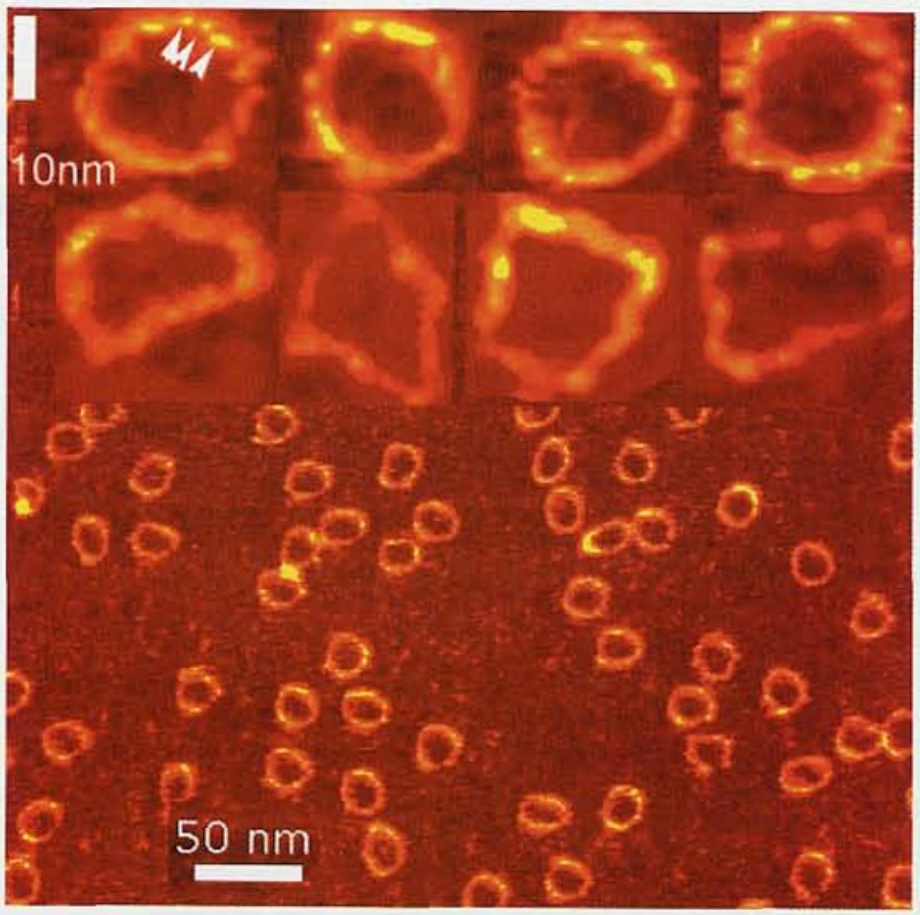

Figure 5: DNA microcircles imaged on mica under buffer solution. Cut-out images are shown at higher magnification above the main image and the arrows point out the $3.4 \mathrm{~nm}$ repeat of the double helix. 


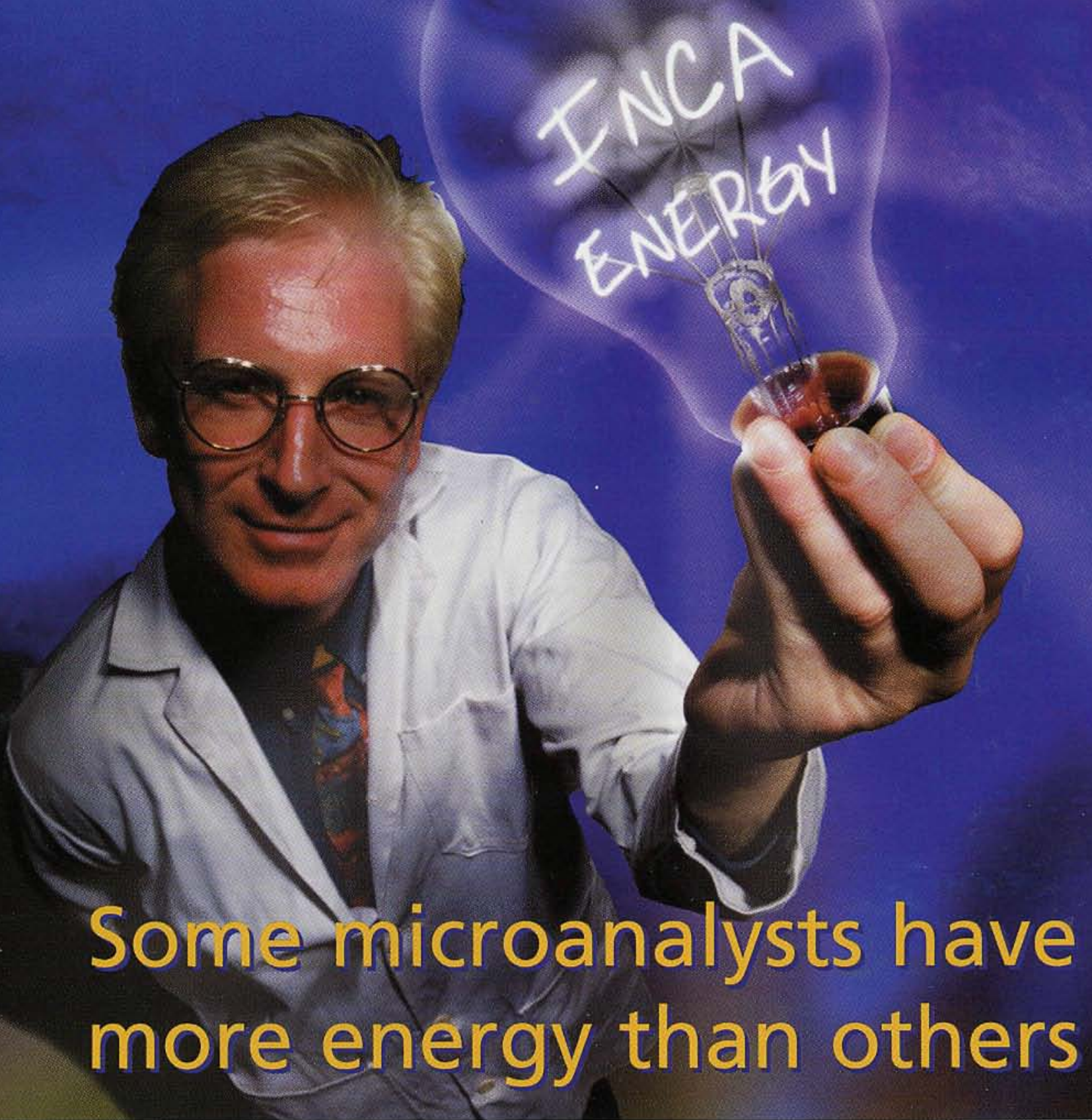

The more power you have - the more you can achieve.

INCAEnergy makes this especially true for today's microanalysts.

INCAEnergy releases the power of Energy Dispersive X-ray (EDX) microanalysis with fast, simple-to-use procedures to provide the most accurate results and significantly boost your microanalysis output.

INCA-power and productivity in microanalysis

For more information on INCA visit our website today at www.oxford-instruments.com/mag

\section{Or call us on:}

USA 1 (978) 3699933 ,

UK +44 (0) 1494442255 ,

France (33)-01-69 852521 ,

Germany (49) 06122 937-176,

INCA is a trademark of Oxford Instruments
Scandinavia (46) 859072550 .

Australia (61) 294846108

Japan (81) 3-5245-3591,

Singapore (65) 337-6848,

China (86) 1068330336.

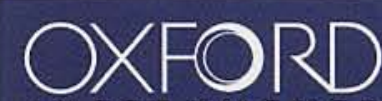

I N S T R U M E N T S 


\section{PROBING THE LIQUID-SOLID INTERFACE WITH DYNAMIC FORCE MICROSCOPY \\ Continued from page 14}

lected features. It is important to measure the width of features across a whole field of images. Selection of isolated 'good' regions is a dangerous procedure because molecular-scale images are inherently noisy. Regions can be found where expected features disappear altogether, which would imply a negative resolution! The highest resolution images of wellisolated DNA molecules were obtained with magnetic-drive DFM, where all the features in the images showed a broadening of little more than $1 \mathrm{~nm}$ (Han, Dlakic et al. 1997). A side-byside comparison of acoustic vs. magnetic drive on the same sample demonstrated substantial extra noise in the images obtained with acoustic drive (Lantz, O'Shea et al. 1994).

It is not clear why the method of drive should influence the resolution in DFM, but it is clear that careful control of oscillation amplitude and approach to the surface are required for high resolution (Lantz, Liu et al. 1998). The smallest possible amplitude and subsequent amplitude reduction are required in order to minimize the interaction between the tip and the sample (Lantz, Liu et al. 1998). On the other hand, the intrinsically noncontact nature of the imaging results in reduced resolution because of the presence of mobile fluid layers between the tip and sample surface. Thus, the optimal operating point needs to be chosen with care. This is hard to do with acoustic drive, because of the background signal in contact and because of the difficulty of calibrating the amplitude of oscillation when the frequency cannot be adjusted at will (Lantz, Liu et al. 1998).

Harder contact appears to result in broader images, possibly because of damage to fine asperities on the tip. This is illustrated in Figure 4 where we show side-by-side DFM images of a small protein (cytochrome c) on a graphite surface under a buffer solution taken with acoustic drive (Figure $4 \mathrm{a}$ ) and under identical conditions with magnetic drive (Figure 4b). We speculate that the difficulty of amplitude calibration in the acoustic case (Lantz, Liu et al. 1998) resulted in rougher contact with the surface and consequently poorer images as illustrated by the insets which show how resolution might be decreased by pushing the tip harder into the surface.

Examples of Imaging in fluids and controlled environments

Figure 5 shows images of synthetic DNA microcircles (Han, Dlakic et al. 1997). These tiny molecules are only 168 bases in length, resulting in closed circles of less than $20 \mathrm{~nm}$ diameter. The average full-width of all the images is $3.5 \mathrm{~nm}$, implying an average broadening of just over a $\mathrm{nm}$. This level of resolution is just adequate for the major groove of the double helix to become apparent as is illustrated in the blown-up images at the top where the helix repeat is marked with arrows.

The technique is of use outside of a strictly fluid environment, and our next example shows imaging as a liquid film is developed. Figure 6 shows the melting of lactose crystals used in aerosol drug-delivery as the ambient humidity was raised from $76 \%$ (Figure $6 a$ ) to $96 \%$ (Figure $6 \mathrm{~b}$ ). These images were taken using the environmental chamber that is an integral part of the PicoSPM from Molecular Imaging (Phoenix, AZ). Some materials, such as liposomes, are only stable in a liquid environment, and Figure 7 shows images of nanometer sized liposomes imaged in buffer. Magnetic DFM permitted gentle and reproducible imaging of these samples for particle sizing.

We end by showing an example of a biological process

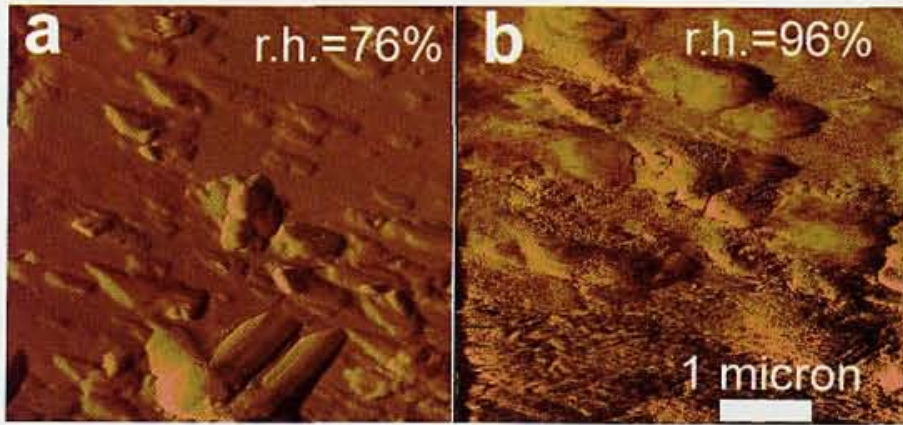

Figure 6: Showing the dissolution of lactose crystals as the relative humidity is raised from $76 \%$ (a) to $96 \%$ (sample courtesy of M. Maniaci and G. Ward of Dura Pharmaceuticals)

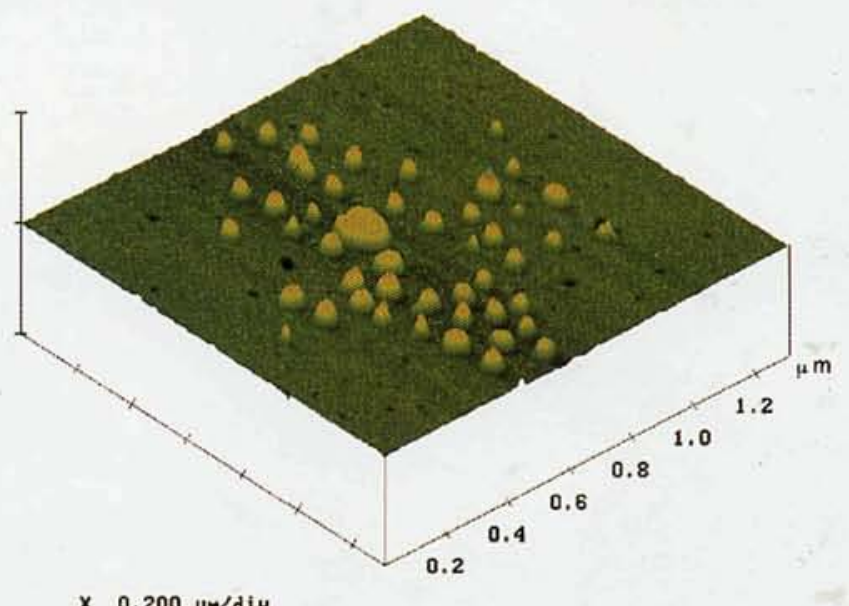

$45,000 \mathrm{~nm} / \mathrm{di}$

Figure 7: Liposomes imaged underwater on a mica surface. (sample courtesy of $R$. Volde of the University of California, San Diego).

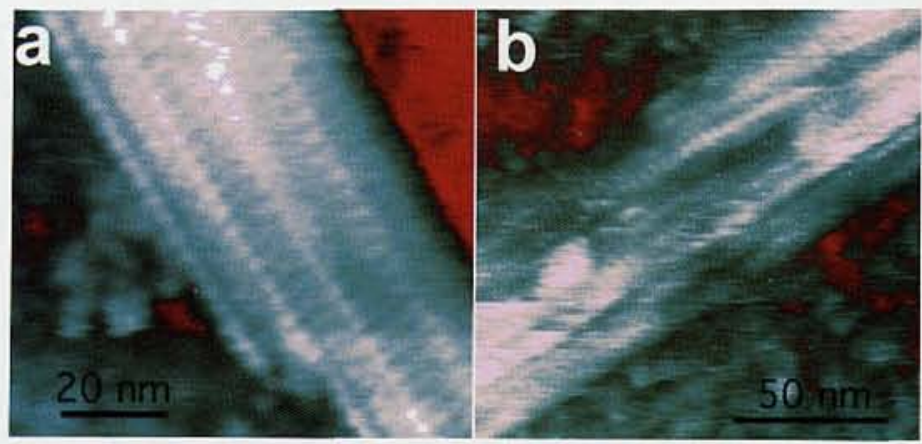

Figure 8: Intact microtubule on mica under buffer (a). When katanin is added (b) digestion of the microtubule is observed (b). (Samples courtesy of R. Vale of the University of California, San Francisco).

imaged at $\mathrm{nm}$ resolution. Figure 8 shows an intact microtubule attached to silanized mica and imaged in microtubule buffer (BRB12 containing $20 \mu \mathrm{M}$ taxol) using magnetic-DFM Thirteen protofilaments are visible across the tubule and each is seen to consist of repeating units of $4 \mathrm{~nm}$ size. These are the tubulin $\mathrm{K}$ and 2 subunits. Such images are easily obtained and give a resolution as good as the best TEM (which is restricted to dried and stained samples). Because the imaging is carried out in buffer solution, it is straightforward to follow processes, and Figure $8 \mathrm{~b}$ shows what happens to these normally stable images on the addition of katanin, an enzyme which digests microtubules. The microtubule is rapidly destroyed during the scan.

Continued on page 18 


\section{Improve Your VPSEM Secondary Imaging Performance With....}

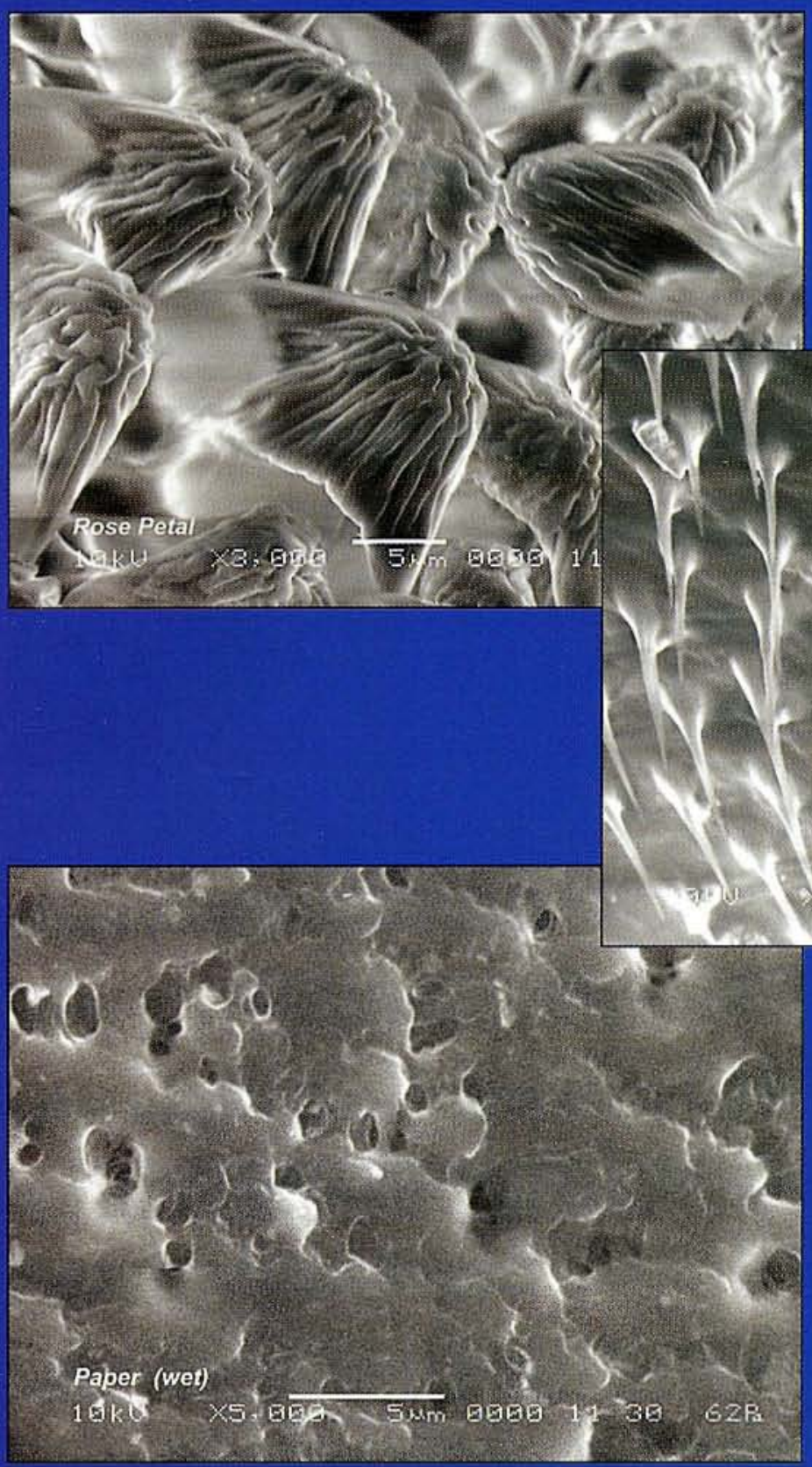

At last-Secondary Electron Imaging for your VPSEM! AMT's new MAXSEM

Detector will enhance the performance of your new or existing instrument.

High Sensitivity

Rapid Scan Rates

Retrofittable To All SEMs
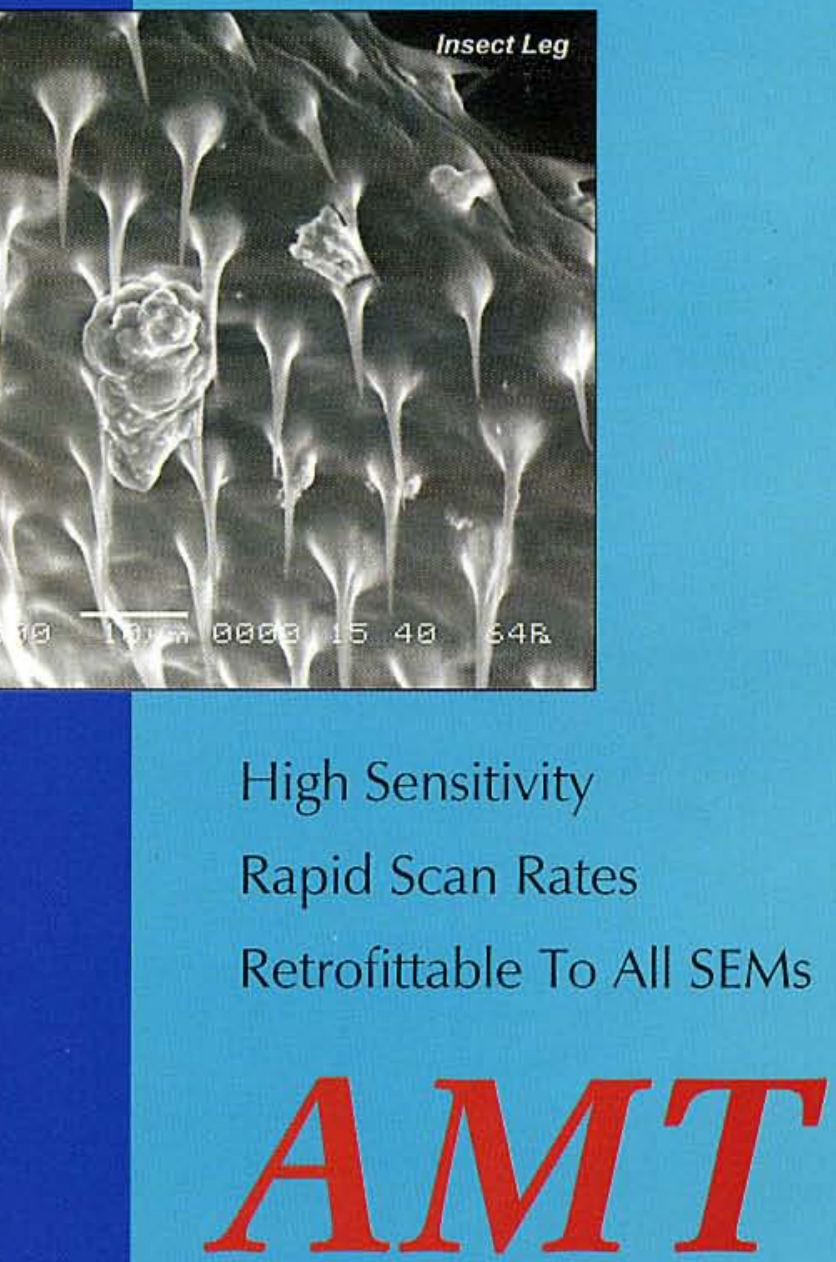


\section{PROBING THE LIQUID-SOLID INTERFACE WITH DYNAMIC FORCE MICROSCOPY \\ Continued from page 16}

\section{Conclusions}

The dynamic force microscope, in which the change in oscillation amplitude of a vibrated tip is detected on approach to a surface, permits gentle non-contact imaging of samples in fluid. It is an important development in realizing the full potential of the atomic force microscope for $\mathrm{nm}$-scale direct imaging of processes at real, chemically active interfaces.

\section{References}

Drake, B., C. B. Prater, A. L. Weisenhorn, S. A. C. Gould, T. R. Albrecht, C. F. Quate, D. S. Cannell, H. G. Hansma and P. K. Hansma (1989). "Imaging Crystals, Polymers, and Processes in Water with the Atomic Force Microscope." Science 243:15861589.

Florin, E. L., M. Radmacher, B. Fleck and H. B. Gaub (1993). "Atomic Force Microscope with magnetic force modulation." Rev. Sci. Instrum. 65: 639-643.

Han, W., M. Dlakic, R. B. Harrington, J. Zhu and S. M. Lindsay (1997). "Strained DNA is kinked by low concentrations of $\mathrm{Zn+."}$ PNAS 94:10565-10570.

Han, W., S. M. Lindsay and T. Jing (1996). "A magneticallydriven oscillating probe microscope for operation in liquids."

App. Phys. Lett. 69: 4111-4114.

Hansma, P. K., J. P. Cleveland, M. Radmacher, D. A. Walters, P. E. Hilner, M. Bezanilla, M. Fritz, D. Vie, H. G. Hansma, C. B. Prater, J. Massie, L. Fukunaga, J. Gurley and V. Eilings (1994). "Tapping mode atomic force microscopy in liquids." Appl. Phys.
Lett. 64: 1738-1740.

Lantz, M., Y. Z. Liu, X. D. Cui, H. Tokurnoto and S. M. Lindsay (1999). "Dynamic force micrsocopy in Fluid." Surface and Interface Analysis: in press.

Lantz, M. A., S. J. O'Shea and M. E. Welland (1994). "Force microscopy imaging in liquids using ac techniques." Appl. Phys. Lett. 65: 409-411.

Lindsay, S. M., Y. L. Lyubchenko, N.J. Tao, Y. Q. Li, P.I. Oden, J. A. DeRose and J. Pan (1993). "Scanning Tunneling Microscopy and Atomic Force Microscopy Studies of Biomaterials at a liquidsolid interface." J. Vac. Sci. Technol. 11: 808-815.

O'Shea, S. J., M. A. Lantz and H. Tokomoto (1999). "Damping near solid-liquid interfaces measured with atomic force micrsocopy." Langmuir: in press.

Putman, C. A. J., K. O. V. d. Werf, B. G. deGrooth, N. F. V. Hulst and J. Greve (1994). "Tapping mode atomic force microscopy in liquid." Appl. Phys. Letts 64: 2454-2456.

Schaffer, T. E., J. P. Cleveland, F. Ohnesorge, D. A. Walters and P. K. Hansma (1996). "Studies of Vibrating Atomic Force Microscope Cantilevers in Liquid." J. Appl.. Phys. 80: 3622-3627.

Figure 6: Showing the dissolution of lactose crystals as the relative humidity is raised from $76 \%$ (a) to $96 \%$ (sample courtesy of M. Maniaci and G. Ward of Dura Pharmaceuticals).

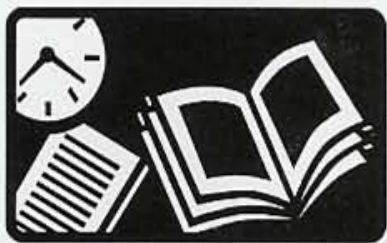

\section{Si(Li)-Detectors}

stainless steel dewar, UHV technology excellent energy resolution

best choice for light element analysis unrivalled long term stability

\section{Microanalysis Systems}

standardless analysis on rough surfaces and particles physical calculation of the bremsstrahlung integrated sexpert system « comprehensive error calculation application-specific automatic quantification

\section{$\triangle N E W$ (xic) Element Imaging} liquid nitrogen free RÖNTEC XFlash detector 8 times faster than EDX with digital pulse processing combines with any existing EDX or imaging system

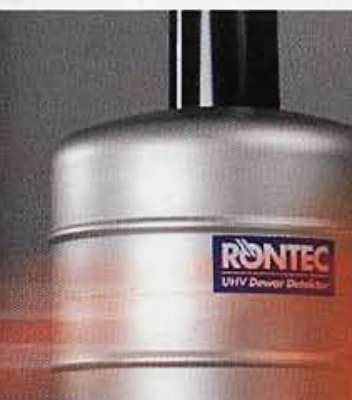

